\title{
Research on The Rapid Development of The Use and Maintenance of Earth Auger
}

\author{
Ming Su \\ Hainan Institute of Science and Technology, Haikou Hainan, 571126, China
}

Keywords: Earth auger, Use, Maintenance.

\begin{abstract}
At the time of the mechanization and returning farmland to forest in China , in order to plant trees as effectively as possible, earth auger as a kind of important mechanical equipment, its function will directly affect the quality and speed of afforestation in our country. Therefore, strengthening the exploration of the use and maintenance of earth auger is very important . This article summarizes the basic characteristics of earth auger, lists the main types and operation mechanism of earth auger, focuses on the usage method and maintenance strategy of earth auger , and looks forward to the future development trend of earth auger.
\end{abstract}

\section{Introduction}

Because of the implementation of the policy of returning farmland to forest and greening policy in recent years and the rapid development of fruit tree planting at the same time, the use of various types of mechanical equipment for afforestation has become the first choice of modern people now, earth auger is this kind of new modern planting tools. This machine is driven by a gasoline engine and has many advantages such as, overall weight is relatively light, operation is more convenient, maintenance is fairly simple, fuel consumption is very low, noise is smaller, very easy to start and operating cost is relatively low. So, now the earth auger has been developed quickly and its range of application is becoming wider and wider.

\section{The basic characteristics of earth auger}

The introduction of earth auger for tree planting activities, can improve the effectiveness of mechanical planting and also ensure the quality of nursery-grown plant. Pit diameter and depth digged by earth auger can well meet the requirements of planting trees , and the pit diameter has a good verticality, pit walls are usually quite regular and not too smooth , therefore it is very good for the growth of root system. Even on the land with low quality, such machines, digging unearthed rate can reach to more than $90 \%$, both adding fertilizer to the pit and topsoil backfill are all very practical. The traditional artificial digging method mainly uses picks planning and shovel digging , which is not only laborious but also quite time consuming and easily lead to operator fatigue, efficiency tends to decrease, after the introduction of digging machine ,for the speed, it is thirty times faster than the artificial . The aiguille of earth auger is usually produced by synthetic manganese steel and appears to be more hard . It can work effectively on hard soil, super hard soil and frozen soil so as to achieve the most desirable results.

\section{The main types of earth auger}

There is a variety of earth auger. If classified by hitching form of matched power, earth augers can be divided as suspension type, portable type, trail-type and self-propelled type. If classified by the shape of the drill machine, the drills can be divided as auger type, spiral belt type, multi-punching, leaf-type and helical teeth type. For the earth auger of suspension type, the machine is usually hung on the tractor, often used in relatively flat terrain, or where the tractor can be passed, the lifting and drop of the drill is mainly manipulated by the tractor driver through hydraulic system, the diameters and the 
depth of the holes are relatively large, and also multiple bits can be operated at the same time. For the earth auger of portable type, this equipment is mainly assembled together with gasoline engine and become into one union, portable type machine is operated by single or double people, it is usually lighter in weight, which is applicable to the complex mountain area and hilly area the where the tractor can't pass through, the diameters and the depth of the holes are relatively small, and it can also be used on fertilizer and stick-buried on fruit trees. For trail-type earth auger, the equipment is usually installed on the small car, pulled through the tractor effectively, hooking up is very convenient, and also can't be restricted by the main structure of tractor, but the defect lies in that the structure is very complex and lack of mobility.

\section{The operation mechanism of earth auger}

Earth auger are generally composed of small gasoline engine, clutch, transfer-box and specially designed drilling tool, usually be suitable for punching in the mountainous region, frozen earth or ice layer, and is widely used in garden, trees planting, road construction and many other fields. Large or medium-sized earth auger usually rotates through pushing the shaft by the port of power-output shaft of tractor, and uses the universal drive shaft and gear box to drive the drill to rotate, so as to make the machine blade can cut the soil, and then the hydraulic efficiency elevation structure of tractor will raise or lower the drill, the chipped out soil or stone powder is put forward through the spiral blade, and is crushed to a decentralized state, which is thrown to the ground around the pit-mouth through the positive role of centrifugal force, so as to realize the whole process of the excavation work.

\section{The usage of earth auger}

The effectiveness of earth auger is closely linked to dig land types, power level and reducing speed of bit. If the bit declining speed is faster, then the power consumption is naturally larger and work efficiency is high; if the bit is declining at a slower speed, then the power consumption is relatively small and the operation efficiency is naturally low. For example, using a medium earth auger to dig a pit about $60 \mathrm{~cm}$ deep, usually need six to seven seconds, and for the more hard earth it requires about twenty seconds.

Specifically, the use of the earth auger can be summarized as the following six aspects: First is the preparation before holes digging. Operation staff should wear thick clothes and wear protective articles such as gloves and helmet protective carefully, and drinking is prohibited before starting the machine. Operation staff must be strictly in accordance with specific operating procedures when using the machine, must never operate a machine with fault or hidden dangers, if there may be a problem of the device, operation staff should stop at the first time, and security maintenance staff should fix it in time. Before starting the work, operation staff should carefully check the connection between power-output shaft of the tractor and the earth auger, once find the bolt connection is loose they should quickly make reinforcement. The second is to do a good job of assembly. Operation staff should properly install the relevant fittings such as the bit, axis pin and cotter pin in accordance with the requirements of machine instructions, check and firmly tighten all the fasteners, all the lubricating points and the rotating parts should be injected with a certain number of lubricating oil, fully debug each part to ensure their smooth operation. Pour gear oil into gearbox, mix gasoline and oil with the proportion of 18:1, and fill the fuel tank after the shake up. Also need to pay attention that fuel filling should not be to much, so as to avoid the oil spill out. The third is to start the earth auger and try to dig. When starting the machine, the operation staff should pull out the choke valve in the gasoline engine, adjust the speed lever to leave away from the position of rest. The left hand should grasp the handle of the rack, the right hand should fully pull the starting rope, when the engine is started, the starting rope can be put back to the original place, then use the right hand to seize the governor rod and pull it slowly, and make sure the speed control rod can return back to the position of rest. Mean while, ensure the auger spindle can be stationary. When choosing the specific digging point, the 
started earth auger should be raised up and its drilling bit can be inserted directly into the soil. In the using process, when the soil is relatively solid or the pit diameter is too large, it can be divided into several times for excavation, firstly, uses small diameter drill bit for excavation, then uses the large diameter drill bit to make the small pit become large, until it reaches to the expected maximizing pit diameter and pit depth. When reaches the digging depth of presupposition, the drill bit can be put forward and the machine can be moved to the next pit. The forth is to realize the diversified choices of the drill of earth auger. When encountered with an area with a very solid soil, multiple step mining method should be adopted .If one time molding can not meet the related requirements, then the concept of multiple molding process can be introduced, which means on the basis of the pit with relatively small diameter for the first time digging, the second time digging can make the pit to meet the requirements. The fifth is to mark the pit. In the process of actual use of earth auger, the driver should always put the control rod of the tractor in a floating state ,therefore to make sure the drilling bit can really aim at the mark on the pit to be digged, and it depends on its own weight to achieve landing. When the drilling bit of earth auger aiming at the mark center of the pit, and then lay it down by its own weight. Power output shaft of tractor can make use of drive shaft and reducer to push the drill bit into the soil. After the drill bit cutting off the soil in the center position, the next step is to cut the soil beneath the blade of the drill bit. The soil under the centrifugal force will be thrown to the surrounding, it will rise to the ground along the spiral blade under the effect of friction and been thrown the around the hole. After digging to the expected depth, operator should also use a hydraulic suspension device to increase the drill bit to the state of the carriage, so as to complete the whole process of digging. The sixth is to use the accelerator reasonably. Once the drill bit with $30-40 \mathrm{~cm}$ diameter of the earth auger is used for the work on hard land, operator should not vigorously step on the accelerator, especially when the drill bit is enter into the $50 \mathrm{~cm}$ of the soil layer. Because the resistance is very large, the engine tends to generate overload conditions, which is easy to cause the drill bit stop rotating. At this time, the whole earth auger should be raised up and spinned out through the stepping on the accelerator and then stop the drill bit.

\section{The maintenance strategy of earth auger}

In order to prolong the service life of earth auger and improve work efficiency as much as possible, strengthening the maintenance work is extremely important, this requires the maintenance of digging machine should be in scientific method. The first is to carefully check the oil level before and after each time of use. Under normal circumstances, probably the engine oil should be replaced after a total of thirty hours working time. The engine oil should genuine products and make sure the environment is clean. The second is to handle the refrigerator scientifically. It should be run for 3 minutes at low speed before it can be properly applied. Even if there is no work for it, there is no need for booming throttle for a long-term, gasoline engines should not run faster than the normal speed except in the case of special emergency response to machine halt based on low speed state of the machine .The third is that after the long hours of work of two stroke air-cooled gasoline engine, its temperature of crank case should not exceed $90{ }^{\circ} \mathrm{C}$. If there are signs of overheating then the machine should be stopped 20 to 15 minutes in time before continuing to work, so as to ensure the gasoline engine, especially the cylinder heat sink part can keep clean. The fourth is that non professional workers can't adjust the carburetor by themselves. If there is a must for the adjustment, they should firstly tighten the oil needle in a clockwise direction, and then return 1.5 turn to the 1.75 turn in the direction of the counter clockwise direction. The fifth is that after each run of 100 to $300 \mathrm{H}$, the operator should use wood or bamboo to clean up carbon deposition which is attached to the cylinder, piston, valve and other parts. Carbon deposition should not enter into the cylinder bore and the valve seat. Meanwhile, filter element of air filter in machine should also be checked and cleaned frequently and replaced according to the specified time. The sixth is that filling of fuel into the earth auger must implement in cooler conditions. Gasoline should be clean. In the process of fuel 
charging, routine check and maintenance, spark plug cap should be removed before implementation, refueling operator shall not smoke and should be far away from the fire source during the operation process. The seventh, for the earth auger with clutch, the clutch should keep exposed, that means machine can not be started before installation completed. Before and after each use of the machine , operators should carefully check the close degree of the main working parts and other parts of the bolt. The eighth is that try as far as possible not to operate earth auger indoor. In the process of machine operation ,in addition to the operator, irrelevant personnel must keep at least 15 meters away from the machine. The ninth is that after the operation , the dirt attach to the tractor and earth auger should be cleaned up quickly, lubricating oil should be injected into the lubricating points, and drill bit spiral blade should be coated with some oil to prevent corrosion . The tenth is that once the machine will not be used for a long time, it should be fully maintained and conserved in the condition of room ventilation and desiccation.

\section{The future development trend of earth auger}

The first is trying to achieve a variety of functions in one. China's afforestation work has distinctive features such as seasonal and regional, machine operating time is relatively short, and the usage rate of the equipment with a single performance is very low, so the design must consider the multi use of one machine and other issues as much as possible in the future, that means the drill bits can be changed to adapt to different types of soil and work environment, at the same time, designer should design the universal frame, ensure that the machine can complete different silvicultural projects and improve the service efficiency after changing the working parts. The second is trying to achieve harmony between man and machine. Designers should further improve the man-machine engineering design system, make efforts to make it conform to the psychological and physiological characteristics of the operator, reduce the operator's psychological pressure as much as possible, and promote the improvement of work efficiency. The third is to expand the scope of usage. The machine should not be only suitable for plain or sandy land, but also should be used in the mountains or in the gully, so as to enhance the applicability of the digging and planting.

\section{Summary}

In conclusion, the use and maintenance of earth auger should be in line with local characteristics and give full play to the effectiveness of afforestation, enhance the work efficiency and holes digging in the maximum and reduce the intensity of labor effectively. Reasonable purchase, use and maintenance of earth auger can significantly improve the working efficiency. For large-scale afforestation area, researchers should focus on the development of large earth auger that not only the production efficiency is high and the automation level is quite high, for small areas, steep slopes and areas with a lot of stones, researchers should focus on the research and development of flexible, small and practical earth auger with strong digging ability.

\section{Acknowledgments}

Project 1: The research and development of earth auger for fruit tree planting with multi cutter tooth, multi gap and cutting earth drill , serial number HNKY2014-109; Project 2: The research and development of earth auger for fruit tree planting with multi blade , multi gap and cutting earth drill , serial number 20155209.

\section{References}

[1] Tong Qinghui. Afforestation effect analysis of the earth auger, Forestry Machinery \& Woodworking Equipment, 2011(9). 
[2] Zhu Zanbin, Li Shusen, Zen Jianfeng. Improved design of the earth auger and modal analysis of bit, Agricultural mechanization research, 2012(2).

[3] Sun Jinjun. Basic structure and purchase principles of earth auger, Farm machinery using \& maintenance,2013(7).

[4] Lu Jian,Miu Ming,Lu Shaoying. Design and test of the Vehicle tree planting earth auger, China Agricultural Chemicals, 2014(6).

[5] Li Lieliu, Xu Yun. Usage technology of tree planting earth auger, Scientific breeding, 2015(4). 